\section{Case Reports in Ophthalmology}

\title{
Epiretinal Membrane in Dome-Shaped Macula Complicated with Serous Retinal Detachment: Transient Efficacy of Surgery
}

\author{
Guillaume Soudier $^{a} \quad$ Alain Gaudric ${ }^{b}$ Vincent Gualino $^{b}$ Mathieu Nardin ${ }^{c}$ \\ Claude Speeg-Schatz ${ }^{c}$ David Gaucher ${ }^{\mathrm{a}}$ \\ aOphtalmology, Nouvel Hôpital Civil, Strasbourg, France; bUniversity Hospital of \\ Lariboisière, University Paris 7, Paris, France; ' Ophthalmology, Hôpitaux Universitaires de \\ Strasbourg, Strasbourg, France
}

\section{Keywords}

Myopia - Dome-shaped macula Epiretinal membrane

\begin{abstract}
Dome-shaped macula (DSM) is an entity recently described as a convex anterior protrusion of the macular area within a posterior myopic staphyloma. Specific complications were associated with DSM, like serous retinal detachment (SRD). We describe a woman presenting with a decreased vision at 20/50. SD-OCT scans were performed, showing a macular bulge. SRD was present and an epiretinal membrane could also be observed. Fluorescein angiography and indocyanin green angiography did not show any leakage nor choroidal neovascularization. Epiretinal membrane peeling was performed, and 3 months after surgery, SRD completely disappeared. However, SRD reappeared 1 year after surgery and enlarged within 2 years following surgery. In conclusion, two mechanisms could be considered for physiopathology of SRD: first, the epiretinal membrane may have exerted traction on the macular retina, second, vitreous body might constitute a tank for cytokines and/or other factors, triggering subretinal fluid accumulation, leading to SRD.

(c) 2017 The Author(s)

Published by S. Karger AG, Basel
\end{abstract}




\section{Case Reports in Ophthalmology}

\section{Introduction}

Dome-shaped macula (DSM) is an entity recently described as a convex anterior protrusion of the macular area within a posterior myopic staphyloma [1]. The original description by Gaucher et al. [1] was made out of a group of highly myopic eyes and some visionthreatening complications were specifically described, such as choroidal neovascularization, macular retinal atrophy, flat irregular pigment epithelium deposits, epiretinal membrane, and serous retinal detachment (SRD). SRD physiopathology still remains unclear. Different hypotheses have been proposed. Viola et al. [2] thought that thickened central macular choroid in DSM could lead to choroidal leakage as reported in central serous chorioretinopathy. Choroidal vascular changes in the central zone and specific abrupt choroidal thickness changes between central and peripheral macula [3] may alter choroidal blood flow and trigger SRD development $[3,4]$.

In this clinical case, we describe a patient presenting with DSM associated with epiretinal membrane and SRD. After epiretinal membrane peeling, transient resolution of SRD was noticed, suggesting that vitreous removal had influenced SRD evolution.

\section{Case Report}

We report a case of a 63-year-old woman presenting with a decreased vision and metamorphopsia. Her best-corrected visual acuity (BCVA) on the right eye was 20/50. The axial length was $24.4 \mathrm{~mm}$ and spherical equivalent was -5 .

The initial OCT horizontal scan showed a macular bulge with a maximal height of 760 $\mu \mathrm{m}$ measured as previously described [5, 6] (Fig. 1). On the vertical scan, there was no macular bulge: the horizontal axis was the most cambered on SD-OCT, indicating that this DSM had a vertical oval-shaped pattern [6]. An SRD was present and an epiretinal membrane could also be observed on both vertical and horizontal OCT scans on Spectralis HRA OCT ${ }^{\circledR}$ (Heidelberg Engineering, Heidelberg, Germany) (Fig. 1).

Fluorescein angiography (FA) and indocyanine green angiography (ICGA) showed hypofluorescent and hypocyanescent perifoveal zones without any leakage or choroidal neovascularization. Infrared image showed multiples epicenters of contraction leading to retinal folds (Fig. 2).

Epiretinal membrane peeling was performed as vision was decreasing since the last control 6 months before. One month after surgery, we observed a progressive decrease of SRD and a complete resolution of subretinal fluid at 3 months (Fig. 3). However, SRD reappeared 1 year after surgery and enlarged within 2 years following surgery (Fig. 3 ).

\section{Discussion}

Several studies showed the presence of SRD in patients presenting with DSM [1, 2, 6-8]. Different hypotheses were advanced to explain SRD appearance. In a robust study, Viola et al. [2] noted that there was more pinpoint leakage on FA and hypercyanescent choroidal points on ICGA in eyes with SRD than without. These findings showed similarities with chronic central serous chorioretinopathy.

In our clinical case, this leakage on FA and ICGA was not present. The fact that vitrectomy and epiretinal membrane peeling could improve transiently the SRD may suggest two 
Case Reports in
Ophthalmology

Case Rep Ophthalmol 2017;8:515-520

DOI: $10.1159 / 000481703$

(c) 2017 The Author(s). Published by S. Karger AG, Basel www.karger.com/cop

Soudier et al.: Epiretinal Membrane in Dome-Shaped Macula Complicated with Serous Retinal Detachment: Transient Efficacy of Surgery

mechanisms for SRD physiopathology: first, the epiretinal membrane may have exerted traction on the macular retina, favoring the apparition of SRD. Second, the vitreous body might constitute a tank for cytokines and/or other factors, triggering subretinal fluid accumulation and finally leading to SRD formation.

Some authors noted a variation of choroidal thickness between peripheral and central macular areas in a case series [3]: they suggested that this difference could increase choroid hydrostatic pressure leading to SRD. However, we did not find significant results in SRD subgroups, in a series comparing choroidal thickness in DSM and no-DSM groups [4]. Furthermore, the different treatments usually used for hematoretinal barrier restoration (i.e., eplerenone, spironolactone, photodynamic therapy, anti-VEGFs, etc.) seemed inefficient, suggesting other mechanisms than external blood barrier disruption [5, 8]. Even if spontaneous resolution has been observed in a certain proportion of cases [5], the sequence of events in this case strongly suggests a role for vitrectomy in the transient disappearance of SRD. This evolution of fluid movements following vitrectomy has already been observed in macular edema pathology [9]. The removal of the vitreous body may have decreased the level of some factors causing SRD. Oxygen and other factors regulating the external blood retinal barrier may then be implicated. Recently, spironolactone has been shown to have sometimes positive effects on SRD in DSM [10]. Aldosterone or other effectors of aldosterone receptors may be present in the vitreous; their removal during vitrectomy might be responsible for transient decrease of SRD in this case.

\section{Conclusion}

SRD is a common complication in DSM, in which treatment management is a difficult task. Vitrectomy seems to play a role for transient resolution of SRD associated with DSM. The mechanisms of this effect are unclear; SRD may be due to the surgical removal of factors triggering subretinal fluid accumulation.

\section{Statement of Ethics}

This study adhered to the principles of the declaration of Helsinki. The ethics committee of the French Society of Ophthalmology did not find any challenges to the medical and scientific rules of ethics as accepted in France.

\section{Disclosure Statement}

The authors have nothing to disclose.

\section{References}

1 Gaucher D, et al: Dome-shaped macula in eyes with myopic posterior staphyloma. Am J Ophthalmol 2008;145:909-914.

2 Viola F, et al: Choroidal findings in dome-shaped macula in highly myopic eyes: a longitudinal study. Am J Ophthalmol 2014;159:44-52.

-3 Deobhakta A, et al: Localized choroidal thickness variation and pigment epithelial detachment in domeshaped macula with subretinal fluid. Ophthalmic Surg Lasers Imaging Retina 2015;46:391-392. 


\section{Case Reports in Ophthalmology}

Soudier et al.: Epiretinal Membrane in Dome-Shaped Macula Complicated with Serous Retinal Detachment: Transient Efficacy of Surgery

Soudier G, et al: Macular choroidal thickness in myopic eyes with and without a dome-shaped macula: a case-control study. Ophthalmologica 2016;236:148-153.

5 Soudier G, et al: Long-term evolution of dome-shaped macula: increased macular bulge is associated with extended macular atrophy. Retina 2016;36:944-952.

-6 Caillaux V, et al: Morphologic characterization of dome-shaped macula in myopic eyes with serous macular detachment. Am J Ophthalmol 2013;156:958-967 e1.

-7 Errera MH, et al: The extended clinical phenotype of dome-shaped macula. Graefes Arch Clin Exp Ophthalmol 2014;252:499-508.

-8 Fernandez-Vega Sanz A, et al: Serous retinal detachment associated with dome-shaped macula and staphyloma edge in myopic patients before and after treatment with spironolactone. J Ophthalmol 2016;2016:8491320.

$\$ 9$ Jackson TL, et al: Pars plana vitrectomy for diabetic macular edema: a systematic review, meta-analysis, and synthesis of safety literature. Retina 2017;37:886-895.

10 Dirani A, et al: Resolution of foveal detachment in dome-shaped macula after treatment by spironolactone: report of two cases and mini-review of the literature. Clin Ophthalmol 2014;8:9991002.
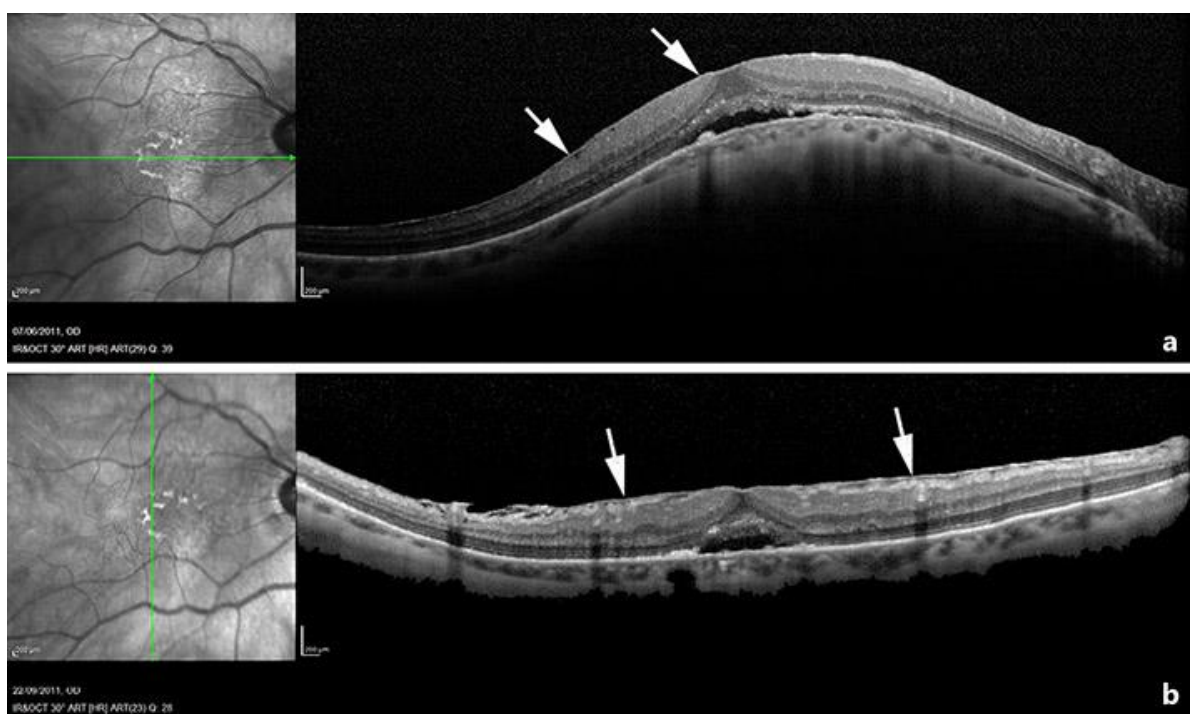

Fig. 1. The initial OCT horizontal scan showed a macular bulge with a maximal height of $760 \mu \mathrm{m}$ measured (a). On the vertical scan, there was no macular bulge: the horizontal axis was the most cambered on SDOCT Spectralis HRA OCT ${ }^{\circledR}$ (Heidelberg Engineering, Heidelberg, Germany), indicating that this DSM had a vertical oval-shaped pattern (b). An SRD was present and an epiretinal membrane could also be observed on both vertical and horizontal OCT scans. 


\section{Case Reports in Ophthalmology}
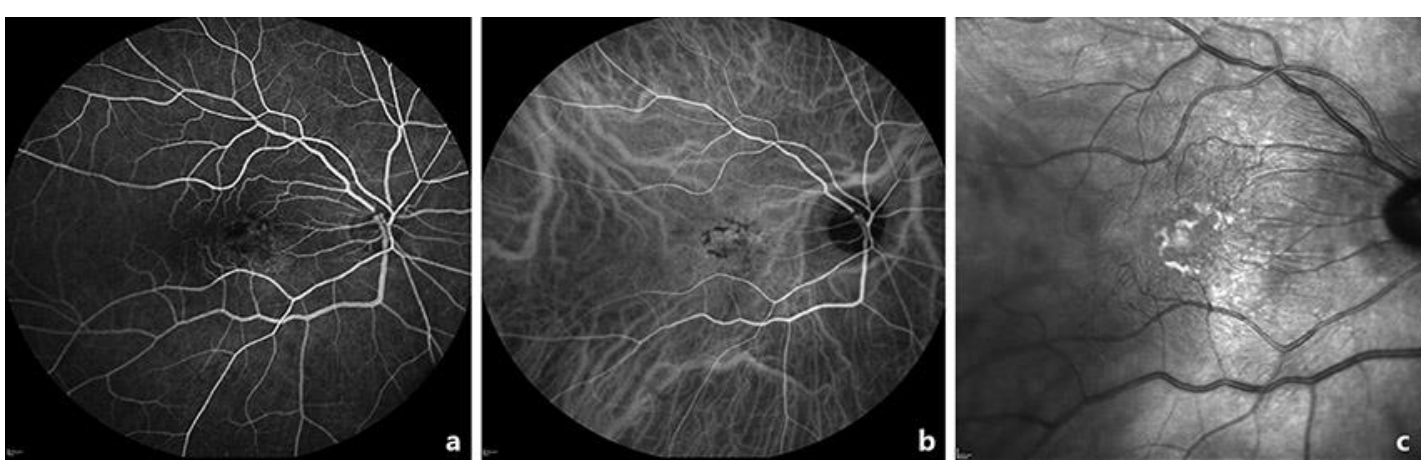

Fig. 2. Both fluorescein angiography (a) and indocyanine green angiography (b) showed hypofluorescent and hypocyanescent perifoveal zones without any leakage or pinpoints. Infrared image showed multiple epicenters of contraction leading to retinal folds (c).

Soudier et al.: Epiretinal Membrane in Dome-Shaped Macula Complicated with Serous Retinal Detachment: Transient Efficacy of Surgery

\section{sis}




\section{Case Reports in Ophthalmology}

Case Rep Ophthalmol 2017;8:515-520

DOI: $10.1159 / 000481703$

(c) 2017 The Author(s). Published by S. Karger AG, Basel www.karger.com/cop

Soudier et al.: Epiretinal Membrane in Dome-Shaped Macula Complicated with Serous Retinal Detachment: Transient Efficacy of Surgery
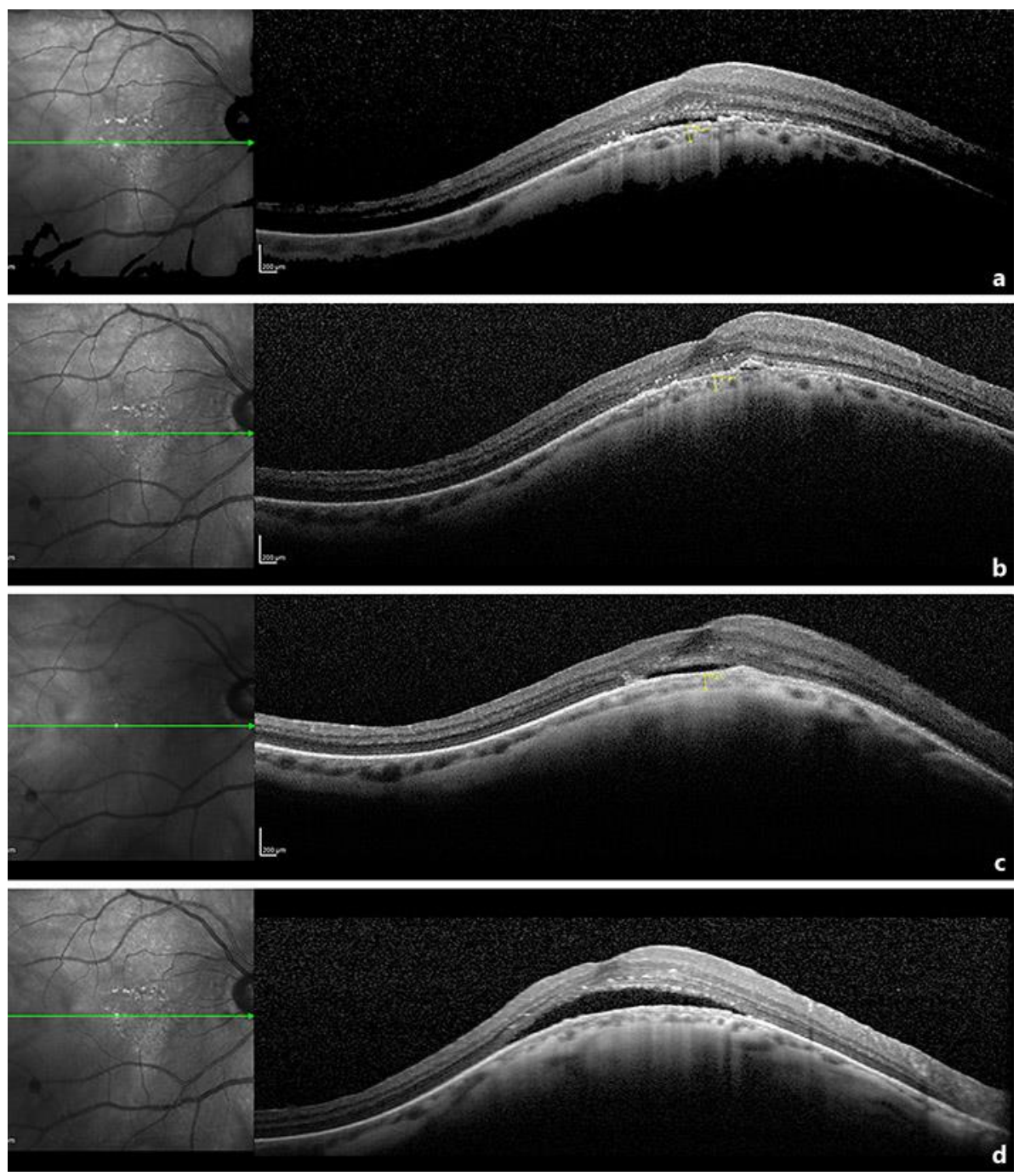

Fig. 3. One month after surgery, we observed a progressive decrease of SRD (a), and a complete resolution of subretinal fluid at 3 months (b). However, SRD reappeared 1 year after surgery (c) and enlarged within 2 years following surgery (d). 